\title{
Semiclassical theory of Fermi resonance between stretching and bending modes in polyatomic molecules
}

\author{
Gregory A. Voth and R. A. Marcus \\ A. A. Noyes Laboratory of Chemical Physics, California Institute of Technology, ${ }^{\text {a) }}$ Pasadena, California 91125
}

(Received 19 November 1984; accepted 14 January 1985)

\begin{abstract}
Approximate semiclassical solutions are developed for a system of a Morse oscillator coupled to a harmonic oscillator via a nonlinear perturbation. This system serves as a model for the interaction of an excited stretching mode with a bending mode in a polyatomic molecule. Three semiclassical methods are used to treat this model. In particular, a matrix diagonalization, a two-state model, and a uniform semiclassical approximation (USC) based on Mathieu functions are each used to determine the splittings and state mixing involved in these stretch-bend Fermi resonances. For small perturbations, approximate analytic semiclassical expressions are obtained for the system treated. These analytic expressions are given for the splittings using a two-state or USC method and for the overlaps of the zeroth order states with the eigenstates of the molecule using a USC method.
\end{abstract}

\section{INTRODUCTION}

The present paper treats the 1:2 resonant interaction of a stretching mode with a bending mode in a polyatomic molecule. This system is modeled classically using a resonance Hamiltonian $^{1-6}$ to describe the nonlinear interaction of a Morse oscillator stretching vibration with a harmonic bending vibration. Several semiclassical methods are used to obtain information about the quantum Fermi resonance resulting from this nonlinear interaction. The results of these methods are compared with each other, with those obtained from a recent uniform semiclassical treatment, ${ }^{7}$ and with the exact quantum results.

The experimental incidence of 1:2 stretch-bend Fermi resonance is widespread, the best known being that in $\mathrm{CO}_{2}{ }^{8}$ Previous discussions of these resonances are many, e.g., Ref. 9, and they have appeared more recently in the local mode literature. ${ }^{10}$ The model of a local mode $\mathrm{C}-\mathrm{H}$ stretch interacting resonantly with bending normal modes has also been proposed as a theoretical explanation for the observed Fermi resonances in $\mathrm{CHD}_{3}{ }^{11,12}$ and for the $\mathrm{C}-\mathrm{H}$ overtone linewidths in benzene. ${ }^{13}$ These Fermi resonances are related to the existence of one (or more) classical resonance conditions. ${ }^{1-6}$ The quantum mechanical implications of an isolat$e d$ classical resonance have been discussed by a number of authors, ${ }^{3,7,14-27}$ including their relation to avoided crossings ${ }^{14,24,25}$ and to Fermi resonances. ${ }^{3,7,14,22}$

A straightforward semiclassical matrix technique is presented in Sec. II to treat Fermi resonant systems. Two other semiclassical methods, namely, a semiclassical twostate solution and a uniform semiclassical approximation (USC), are also formulated later in Sec. IV that are based on an effective classical resonance Hamiltonian for the nonlinear interaction of a Morse with a harmonic oscillator (Sec. III). These latter two methods may be used to calculate analytically the splittings between the eigenstates of the system and the overlaps of the zeroth order wave functions with the actual eigenfunctions. The three semiclassical methods are applied in Sec. $\mathrm{V}$ to a model of a $\mathrm{C}-\mathrm{H}$ stretching local mode

\footnotetext{
Contribution No. 7062 .
}

interacting resonantly with a bending mode in a dihalomethane molecule. The results of these calculations, and those obtained using the method of Ref. 7, are discussed in Sec. VI, and concluding remarks are given in Sec. VII.

\section{SEMICLASSICAL MATRIX DIAGONALIZATION}

The classical Hamiltonian for a coupled Morse and harmonic oscillator may be written in action-angle variables as ${ }^{7}$

$$
\begin{aligned}
& H\left(I_{1}, I_{2}, \theta_{1}, \theta_{2}\right) \\
& \quad=I_{1} \omega_{1}^{0}-I_{1}^{2} \omega_{1}^{0} \chi+I_{2} \omega_{2}^{0}+V\left(I_{1}, I_{2}, \theta_{1}, \theta_{2}\right),
\end{aligned}
$$

where $I_{1}$ and $I_{2}$ are the action variables ${ }^{28}$ for the Morse and harmonic oscillators, respectively. $\theta_{1}$ and $\theta_{2}$ are the angles conjugate to $I_{1}$ and $I_{2}, \omega_{1}^{0}$ and $\omega_{2}^{0}$ are the zeroth order harmonic angular frequencies of the Morse and harmonic oscillators, respectively, $\omega_{1}^{0} \chi$ is the anharmonicity of the Morse oscillator, and $V\left(I_{1}, I_{2}, \theta_{1}, \theta_{2}\right)$ is the perturbation. ${ }^{29}(\hbar$ is set equal to 1 throughout the present paper.)

One can use a semiclassical matrix treatment ${ }^{30}$ of the Fermi resonance problem for the Hamiltonian (2.1) in action-angle variables using, as a basis, the semiclassical wave functions ${ }^{25,31}$

$$
\Psi_{n_{1}, n_{2}}^{(0)}=\frac{1}{2 \pi} \exp \left[i\left(n_{1} \theta_{1}+n_{2} \theta_{2}\right)\right]
$$

for the angle representation of the zeroth order states $\left|n_{1}, n_{2}\right\rangle$, where $\left|n_{1}\right\rangle$ and $\left|n_{2}\right\rangle$ are the zeroth order eigenfunctions for the Morse and harmonic oscillators, respectively. The semiclassical action and angle operators ${ }^{25,31} \widehat{I}_{k}$ and $\hat{\theta}_{k}$ in the angle representation acting on these wave functions yield

$$
\widehat{I}_{k} \mid \Psi_{n_{1}, n_{2}}^{(0)}=I_{k} \Psi_{n_{1}, n_{2}}^{(0)} \text { and } \hat{\theta}_{k} \Psi_{n_{1}, n_{2}}^{(0)}=\theta_{k} \Psi_{n_{1}, n_{2}}^{(0)},
$$

where $I_{k}$ and $\theta_{k}$ are the classical variables. The $I_{k}$ are related to the quantum numbers $n_{k}$ by $I_{k}=n_{k}+\frac{1}{2}$ in the case of an oscillator, be it Morse or harmonic.

The semiclassical wave functions are useful because the matrix elements of the perturbation have the form of Fourier components

$V_{l m}(\mathbf{I})=\frac{1}{(2 \pi)^{2}} \int_{0}^{2 \pi} \int_{0}^{2 \pi} V(\mathbf{I}, \boldsymbol{\theta}) e^{-i\left(l \theta_{1}+m \theta_{2}\right)} d \theta_{1} d \theta_{2}$ 
If, e.g., the resonant interaction of the three states $|n, 0\rangle$, $|n-1,2\rangle$, and $|n-2,4\rangle$ is considered, the Hamiltonian represented in this restricted basis has the following diagonal element $H_{i i}$ for the zeroth order state $i$ :

$$
\begin{aligned}
H_{i i}= & \left(n_{1}^{i}+\frac{1}{2}\right) \omega_{1}^{0}-\left(n_{1}^{i}+\frac{1}{2}\right)^{2} \omega_{1}^{0} \chi \\
& +\left(n_{2}^{i}+\frac{1}{2}\right) \omega_{2}^{0}+V_{00}\left(I_{1}^{i}, I_{2}^{i}\right),
\end{aligned}
$$

where $\left(n_{1}^{i}, n_{2}^{i}\right)=(n, 0),(n-1,2)$, or $(n-2,4)$. The diagonal perturbation term $V_{00}$ is the $(l=0, m=0)$ Fourier component [Eq. (2.4)] evaluated at the actions $I_{1}^{i}=\left(n_{1}^{i}+\frac{1}{2}\right)$, $I_{2}^{i}=\left(n_{2}^{i}+\frac{1}{2}\right)$. The off-diagonal perturbation terms $H_{i j}$ between the states $i$ and $j$ have the form

$$
\begin{aligned}
H_{i j}= & \frac{1}{(2 \pi)^{2}} \int_{0}^{2 \pi} \int_{0}^{2 \pi} e^{-i\left(n_{1}^{i} \theta_{1}+n_{2}^{i} \theta_{2}\right)} \\
& \times V(\hat{\mathbf{I}}, \hat{\boldsymbol{\theta}}) e^{i\left(n^{j} \theta_{1}+n_{2}^{j} \theta_{2}\right)} d \theta_{1} d \theta_{2}
\end{aligned}
$$

which is the Fourier component $V_{l m}\left(I_{1}^{j}, I_{2}^{j}\right)$ of the perturbation with $l=n_{1}^{i}-n_{1}^{j}$ and $m=n_{2}^{i}-n_{2}^{j}$. These semiclassical matrix elements are approximate and usually not exactly Hermitian since $H_{i j}=V_{l m}\left(I_{1}^{j}, I_{2}^{j}\right) \neq V_{l m}\left(I_{1}^{i}, I_{2}^{i}\right)=H_{j i}^{*}$. These matrix elements may be made Hermitian in an $a d$ hoc way by evaluating them at any fixed value of the actions [e.g., at the resonance center $\left.\left(I_{1}^{r}, I_{2}^{r}\right)\right]$ or at an intermediate value of the actions between any pair of states $i$ and $j$, using, for instance, the arithmetic mean $\left(I_{k}^{i}+I_{k}^{j}\right) / 2$, where $(k=1,2) .{ }^{32}$ The latter approximation has been used successfully in the calculation of transition dipole matrix elements for Morse ${ }^{33}$ and other ${ }^{34}$ oscillators and has been employed in a description of isolated avoided crossings. ${ }^{24}$ Semiclassical expressions for the matrix elements are frequently simpler to compute than the corresponding quantum mechanical ones. The resulting semiclassical matrix may then be diagonalized numerically to find the eigenvalues and eigenvectors as is done for standard quantum mechanical matrices.

Comparison of the method presented in this section with exact quantum results are given later in Sec. V. In Sec. IV, we formulate a different semiclassical method based on a classical analysis and an effective Hamiltonian given in the next section.

\section{THEORY: CLASSICAL RESONANCE TREATMENT}

\section{A. Treatment of the perturbation}

The perturbation of these coupled oscillator systems is, as usual, ${ }^{1-6}$ expanded in a complex-valued Fourier series

$$
V\left(I_{1}, I_{2}, \theta_{1}, \theta_{2}\right)=\sum_{l=-\infty}^{\infty} \sum_{m=-\infty}^{\infty} V_{l m}\left(I_{1}, I_{2}\right) e^{i\left(l \theta_{1}+m \theta_{2}\right)},
$$

where $V_{l m}\left(I_{1}, I_{2}\right)$ is given by Eq. (2.4). The $V_{00}\left(I_{1}, I_{2}\right)$ Fourier component is the analog of a quantum mechanical "diagonal" first order perturbation correction. These corrections have been discussed previously ${ }^{11,13,35,36}$ within the context of stretch-bend interactions in molecules and are found to modify the zeroth order oscillator frequencies, sometimes appreciably. To include the effect of this perturbation, a new zeroth order Hamiltonian may be defined as

$$
H^{(0)}\left(I_{1}, I_{2}\right)=I_{1} \omega_{1}^{0}-I_{1}^{2} \omega_{1}^{0} \mathcal{\chi}+I_{2} \omega_{2}^{0}+V_{00}\left(I_{1}, I_{2}\right) \text {. }
$$

(This Hamiltonian is the same as that in Ref. 7, except that the $V_{00}$ term is taken as a constant there, namely, its value at certain zeroth order "resonant actions" described later in the Results section.)

Hamilton's equations for the angle variables, based on $H_{0}$, yield

$$
\begin{aligned}
& \dot{\theta}_{1}=\omega_{1}\left(I_{1}, I_{2}\right)=\omega_{1}^{0}-2 I_{1} \omega_{1}^{0} \chi+\partial V_{00}\left(I_{1}, I_{2}\right) / \partial I_{1}, \\
& \dot{\theta}_{2}=\omega_{2}\left(I_{1}, I_{2}\right)=\omega_{2}^{0}+\partial V_{00}\left(I_{1}, I_{2}\right) / \partial I_{2}
\end{aligned}
$$

and that $I_{1}$ and $I_{2}$ are constants of the motion for $H_{0}$ since this zeroth order Hamiltonian contains no angle variables. Since $I_{1}$ and $I_{2}$ are functions of $E_{1}^{0}$ and $E_{2}^{0}$ (the zeroth order energies of the Morse and harmonic oscillators, respectively), Eqs. (3.3) and (3.4) may be rewritten as

$$
\begin{aligned}
& \omega_{1}\left(I_{1}, I_{2}\right)=\left(\omega_{1}^{0}-2 I_{1} \omega_{1}^{0} \chi\right) f, \\
& \omega_{2}\left(I_{1}, I_{2}\right)=\omega_{2}^{0} g,
\end{aligned}
$$

where

$$
\begin{aligned}
& f=\left[1+\partial V_{00}\left(E_{1}^{0}, E_{2}^{0}\right) / \partial E_{1}^{0}\right], \\
& g=\left[1+\partial V_{00}\left(E_{1}^{0}, E_{2}^{0}\right) / \partial E_{2}^{0}\right] .
\end{aligned}
$$

When the angle-dependent perturbation terms from Eq. (3.1) are included in the equations of motion, the actions $I_{1}$ and $I_{2}$ are no longer constants of the motion. In the presence of a nonlinear resonance, these actions slowly oscillate near or about their value at the resonance center ${ }^{1-6}\left(I_{1}^{r}, I_{2}^{r}\right)$. If the factors $f$ and $g$ in Eqs. (3.5) and (3.6) are approximated as the "average" constants $f^{r}$ and $g^{r}$ [given by Eq. (3.7) evaluated at the resonance center], one obtains an effective zeroth order Hamiltonian

$$
H_{0}\left(I_{1}, I_{2}\right)=I_{1} \omega_{1}-I_{1}^{2} \omega_{1} \chi+I_{2} \omega_{2},
$$

where $\omega_{1}, \omega_{1} \chi$, and $\omega_{2}$ denote $\omega_{1}^{0} f^{r}, \omega_{1}^{0} \chi f^{r}$, and $\omega_{2}^{0} g^{r}$, respectively. These effective oscillator parameters are the classical analog to those obtained empirically from the analysis of experimental absorption spectra (cf. discussion in Refs. $11,13,35$, and 36).

The particular classical resonance to be examined here is the $1: 2$ resonance defined by the condition

$$
\omega_{1}\left(I_{1}^{r}\right) \simeq 2 \omega_{2},
$$

where

$$
\omega_{1}\left(I_{1}^{r}\right)=\left(\partial H_{0} / \partial I_{1}\right)_{I_{1}=I_{1}^{r}}=\omega_{1}-2 I_{1}^{r} \omega_{1} \chi
$$

is the (modified) nonlinear angular frequency of the Morse oscillator at the center of the resonance. The use of these modified oscillator frequencies yields values for the resonant actions different from those predicted by the zeroth order Hamiltonian alone [Eq. (2.1) with $V=0$ ]. An accurate approximate method for finding the values of the resonant actions $I_{1}^{r}$ and $I_{2}^{r}$, a nonlinear problem, is given in Appendix A.

The resonance condition (3.9) prompts a canonical transformation of the zeroth order action-angle variables in which there is now a "slow" variable $\alpha$ (low frequency coordinate) ${ }^{1-7}$ :

$$
\begin{aligned}
& 2 \alpha=\theta_{1}-2 \theta_{2}+\delta, \quad \beta=\theta_{2}, \\
& I_{\alpha}=2 I_{1}, \quad I_{\beta}=2 I_{1}+I_{2} .
\end{aligned}
$$

The $\delta$ in Eq. (3.11) is chosen to simplify later the final expression (3.16) and to make the canonical transformation used in 
Ref. 7 suitable for any general perturbation. ${ }^{29}$

The transformation (3.12) applied to Eq. (3.8) yields the effective zeroth order Hamiltonian in the new action variables $I_{\alpha}$ and $I_{\beta}$ as

$$
H_{0}\left(I_{\alpha}, I_{\beta}\right)=I_{\alpha}^{\prime} \Omega-\omega_{1} \chi^{\prime} I_{\alpha}^{2}+I_{\beta} \omega_{2},
$$

where

$$
\Omega=\left(\omega_{1}-2 \omega_{2}\right) / 2, \chi^{\prime}=\chi / 4 .
$$

As is standard in the theory of classical resonances, ${ }^{1-6}$ all highly oscillating terms in the Fourier expansion (3.1) are omitted, leaving the terms appropriate to the $1: 2$ resonance of the form $\exp \left[ \pm i k\left(\theta_{1}-2 \theta_{2}\right)\right]$, where $k$ equals $(1,2, \ldots)$, in addition to the $V_{00}$ term discussed earlier. As usual, ${ }^{1-6}$ only the lowest nonzero $k$ term is then retained, allowing Eq. (3.1) to be written approximately as

$$
V\left(I_{1}, I_{2}, \theta_{1}, \theta_{2}\right) \simeq 2 \operatorname{Re}\left\{V_{1-2}\left(I_{1}, I_{2}\right) e^{i\left(\theta_{1}-2 \theta_{2}\right)}\right\}
$$

when the lowest term is $k=1$. (In other cases, the $k=1$ term may be zero and a term for $k>1$ is required.) Since the perturbation in Eq. (2.1) is real, the relation $V_{l m}^{*}=V_{-1-m}$ was used in obtaining Eq. (3.15). If $2 V_{1-2}$ is written as $V_{0} e^{i \gamma}$, with $V_{0} \equiv 2\left|V_{1-2}\right|$, then Eq. (3.15) may be written as

$$
V \simeq-V_{0} \cos 2 \alpha
$$

when one chooses $\delta$ in Eq. (3.11) to equal $\gamma+\pi$ to obtain the negative sign in Eq. (3.16).

\section{B. Classical resonance Hamiltonian}

Equations (3.13) and (3.16), evaluated at the resonant actions $\left(I_{1}^{r}, I_{2}^{r}\right)$, yield a resonance Hamiltonian ${ }^{1-6}$ for the $1: 2$ resonance $^{37}$

$$
H_{R}=\omega_{1} \chi^{\prime} I_{\alpha}^{2}-I_{\alpha} \Omega+V_{0} \cos 2 \alpha=E_{R} .
$$

The total Hamiltonian $H$ in Eq. (2.1) is then approximately given by

$$
H_{\text {eff }}=I_{\beta} \omega_{2}-H_{R},
$$

which will henceforth be termed the "effective" Hamiltonian. Since $I_{\beta}$ is a constant of the motion in this approximation, $I_{\beta} \omega_{2}$ is a constant. The phase plane behavior of Eq. (3.17) is discussed in Appendix B.

From Eqs. (3.9), (3.10), and (3.14), the resonant action $I_{1}^{r}$ is given by $\Omega / \omega_{1} \chi$. Since, semiclassically, $I_{1}$ equals $\left(n_{1}+\frac{1}{2}\right)$, a resonant Morse "quantum number" $n_{1}^{r}$ may be defined by

$$
n_{1}^{r}=\left(\Omega / \omega_{1} \chi\right)-\frac{1}{2} .
$$

In general, $n_{1}^{r}$ is not an integer.

The two-state and uniform approximation solutions introduced in the next section are based on the effective Hamiltonian (3.18).

\section{THEORY: SEMICLASSICAL METHODS BASED ON $H_{\text {orf }}$}

\section{A. Two-state solution}

Introduction of the action operator ${ }^{25,31} \widehat{I}_{\alpha}=(1 / i) d /$ $d \alpha+1$ into the classical resonance Hamiltonian [Eq. (3.17)] yields the semiclassical Schrödinger equation ${ }^{7}$

$$
\frac{d^{2} \psi}{d \alpha^{2}}-2 i(\xi-1) \frac{d \psi}{d \alpha}+[A-2 q \cos 2 \alpha] \psi=0,
$$

with $\alpha$ being in the interval $(0, \pi)$ and

$$
\begin{aligned}
& \xi=2 \Omega / \omega_{1} \chi=2 n_{1}^{r}+1, \\
& A=\left[4\left(\Omega+E_{R}\right) / \omega_{1} \chi\right]-1, \\
& q=2 V_{0} / \omega_{1} \chi .
\end{aligned}
$$

For the model oscillator system considered in the present paper, the near 1:2 resonance condition can lead to a near degeneracy between the zeroth order states $|n, 0\rangle$ and $|n-1,2\rangle$ for some value of $n$. In many cases of experimental interest, these two states may be the most important states involved in a Fermi resonance. For this reason, and because it is desirable to obtain a simple analytic solution, a two-state solution for Eq. (4.1) of the form

$$
\psi \simeq a \psi_{n}^{(0)}+b \psi_{n-1}^{(0)}
$$

is considered first, where $a$ and $b$ are constants to be determined and where $\psi_{n}^{(0)}$ equals $\pi^{-1 / 2} \exp [2 i n \alpha]$ and $\psi_{n-1}^{(0)}$ equals $\pi^{-1 / 2} \exp [2 i(n-1) \alpha]$ (e.g., Refs. 25 and 31$)$. When Eq. (4.1) is diagonalized in this basis, the eigenvalues are given by

$$
A^{ \pm}=A_{0} \pm \frac{1}{2}\left(4 q^{2}+d^{2}\right)^{1 / 2},
$$

where

$$
\begin{aligned}
& A_{0}=-\left[4\left(n_{1}^{r}\right)^{2}-v^{2}(n)+2 v(n)-2\right], \\
& d=4[1-v(n)], v(n)=2\left(n-n_{1}^{r}\right),
\end{aligned}
$$

and $q$ is given in Eq. (4.2). In terms of Eq. (3.19), this $v(n)$ is twice the "distance" of the eigenstate with quantum number $n_{1}=n$ from the center of the resonance $n_{\mathrm{t}}^{r}$.

The approximate splitting $\Delta E$ of these two eigenstates by the Fermi resonance is therby found from Eqs. (4.2) and (4.4)-(4.6) to be

$$
\Delta E=\omega_{1} \chi^{\prime}\left(4 q^{2}+d^{2}\right)^{1 / 2}
$$

and the two approximate eigenfunctions are ${ }^{38}$

$$
\begin{aligned}
& \psi^{+}=a \psi_{n}^{(0)}+b \psi_{n-1}^{(0)}, \\
& \psi^{-}=-b \psi_{n}^{(0)}+a \psi_{n-1}^{(0)},
\end{aligned}
$$

where

$$
\begin{aligned}
& a=[(\Gamma+d) / 2 \Gamma]^{1 / 2}, \\
& b=[(\Gamma-d) / 2 \Gamma]^{1 / 2}, \\
& \Gamma=\left(4 q^{2}+d^{2}\right)^{1 / 2} .
\end{aligned}
$$

Calculations employing Eqs. (4.7)-(4.10) involve determining $n_{1}^{r}$ from Eq. (3.19) and $q$ from Eq. (4.2), both of which are readily obtained.

The two-state solution presented in this section is not, in general, the same as a semiclassical $2 \times 2$ matrix diagonalization using the method of Sec. II. The difference between these two methods results from the use in the present section of a resonance Hamiltonian (3.17) based on the effective Hamiltonian (3.18), while the method of Sec. II is based directly on the original Hamiltonian (2.1).

\section{B. Uniform semiclassical approximation (USC)}

A uniform approximation for the present coupled oscillator system is obtained by converting Eq. (4.1) to the stan- 
dard Mathieu equation. ${ }^{7,25}$ A function $F(\alpha)$, defined by ${ }^{25}$

$$
F(\alpha)=\exp [i(1-\xi) \alpha] \psi(\alpha),
$$

is used for this purpose: It satisfies Mathieu's equation ${ }^{39(a)}$

$$
\frac{d^{2} F(\alpha)}{d \alpha^{2}}+\left[a_{v}-2 q \cos 2 \alpha\right] F(\alpha)=0,
$$

where

$$
a_{v}=(\xi-1)^{2}+A=4\left[\left(\Omega / \omega_{1} \chi\right)^{2}+E_{R} / \omega_{1} \chi\right],
$$

and $v$ is the order of the Mathieu equation. ${ }^{39(a), 40}$ Equation (4.13) is rearranged to give the energy of the rotor Hamiltonian $H_{R}$ as

$$
E_{R}=a_{\nu} \omega_{1} \chi^{\prime}-\left(n_{1}^{r}+\frac{1}{2}\right) \Omega .
$$

Semiclassical expressions for the Fermi resonance splittings between the eigenstates of the Hamiltonian may be obtained from Eq. (4.14). In general, one is concerned with a resonant progression of nearly degenerate zeroth order states $\left|n_{1}, n_{2}\right\rangle$ for the zeroth order Hamiltonian $(2.1):|n, 0\rangle$, $|n-1,2\rangle,|n-2,4\rangle$, etc. The action $I_{\beta}$ from Eq. (3.12) semiclassically equals $2 n_{1}+n_{2}+3 / 2$ and remains constant along this progression. For any two states of orders $v_{1}$ and $v_{2}$ involved in this resonant progression, the splittings are given from Eq. (4.14) as

$$
\left|\Delta E_{1-2}\right|=\left|a_{v_{1}}-a_{v_{2}}\right| \omega_{1} \chi^{\prime},
$$

where $^{40}$

$$
v_{i} \equiv v\left(n_{1}^{(i)}\right)=2\left(n_{1}^{(i)}-n_{1}^{r}\right) \quad(i=1,2) .
$$

For example, one uses $n_{1}^{(1)}=n$ and $n_{1}^{(2)}=n-1$ in calculating the splitting between the nearly degenerate states $|n, 0\rangle$ and $|n-1,2\rangle$. The characteristic values $a_{v}$ of the Mathieu equation may be obtained from expansions ${ }^{39(b)}$ (if $q$ is small), from tables, ${ }^{41,42}$ or from semiclassical phase integral arguments. ${ }^{43}$

When the relevant dipole moment operator is assumed to be a function of the $\mathrm{C}-\mathrm{H}$ stretching coordinate only, the overlap (squared) of the zeroth order pure local mode state $|n, 0\rangle$ with the actual eigenstates describes the Fermi resonance intensity sharing, ${ }^{11,13,35,44}$ and so is of interest. When $q$ is small enough, an expansion ${ }^{39(c), 42}$ for the solution $F(\alpha)$ of the Mathieu equation (4.12) may be used. From the definition of the wave function $\psi(\alpha)$ in Eq. (4.11), and using Eq. (4.2) for $\xi$ and the expression for $v$ in Ref. 40, the unnormalized wave function may be written as

$$
\begin{gathered}
\psi_{n_{1}}(\alpha) \sim e^{2 i n_{1} \alpha}-\frac{q}{4}\left[\frac{e^{2 i\left(n_{1}+1\right) \alpha}}{\left[v\left(n_{1}\right)+1\right]}-\frac{e^{2 i\left(n_{1}-1\right) \alpha}}{\left[v\left(n_{1}\right)-1\right]}\right] \\
+\frac{q^{2}}{32}\left[\frac{e^{2 i\left(n_{1}+2\right) \alpha}}{\left[v\left(n_{1}\right)+1\right]\left[v\left(n_{1}\right)+2\right]}\right. \\
\left.+\frac{e^{2 i\left(n_{1}-2\right) \alpha}}{\left[v\left(n_{1}\right)-1\right]\left[v\left(n_{1}\right)-2\right]}\right]+\cdots
\end{gathered}
$$

Equation (4.17) properly normalized (cf. Appendix C) yields the following formulas for the overlaps of the zeroth order $|n, 0\rangle$ local mode state with the eigenstates of $|n, 0\rangle$, $|n-1,2\rangle$, and $|n-2,4\rangle$ parentage:

$\int_{0}^{\pi} \psi_{n}^{(0) *}(\alpha) \psi_{n}(\alpha) d \alpha=\sqrt{\pi} N_{n}$,

$$
\int_{0}^{\pi} \psi_{n}^{(0) *}(\alpha) \psi_{n-1}(\alpha) d \alpha=-\sqrt{\pi} N_{n-1}\left[\frac{q}{4[v(n-1)+1]}\right]
$$

and

$$
\begin{aligned}
& \int_{0}^{\pi} \psi_{n}^{(0) *}(\alpha) \psi_{n-2}(\alpha) d \alpha \\
& =\sqrt{\pi} N_{n-2}\left[\frac{q^{2}}{32[v(n-2)+1][v(n-2)+2]}\right] .
\end{aligned}
$$

Here, $\psi_{n}^{(1)}(\alpha)$ denotes $\pi^{-1 / 2} \exp [2 i n \alpha]$ and is the properly normalized zeroth order local mode state $|n, 0\rangle$, and $\psi_{m}$ $(m=n, n-1, n-2)$ is the approximate normalized eigenstate based on Eq. (4.17). It is also of interest to determine the overlap of the zeroth order state $|n-2,4\rangle$ with the eigenstate $\psi_{n-1}(\alpha)$. This overlap is given by

$$
\int_{0}^{\pi} \psi_{n-2}^{(0) *}(\alpha) \psi_{n-1}(\alpha) d \alpha=\sqrt{\pi} N_{n-1}\left[\frac{q}{4[v(n-1)-1]}\right],
$$

where $\psi_{n-2}^{(0)}$ denotes $\pi^{-1 / 2} \exp [2 i(n-2) \alpha]$. At or very close to the actual avoided crossing point between two states, some of overlaps in Eqs. (4.18)-(4.21) are not suitable for computation and a different expression is used. ${ }^{45}$

\section{APPLICATION}

\section{A. The model Hamiltonian}

As an application, we consider a model Hamiltonian for a single $\mathrm{C}-\mathrm{H}$ stretch interacting with a bending mode involving the $\mathrm{C}-\mathrm{H}$ bond in a dihalomethane molecule. In curvilinear coordinates, ${ }^{46}$ such a Hamiltonian may be phenomenologically written as ${ }^{46(a)}$

$$
\begin{aligned}
H= & \frac{1}{2 \mu} P_{R}^{2}+D[1-\exp (-a R)]^{2} \\
& +\frac{1}{2}\left(P^{2}+\omega_{2}^{02} Q^{2}\right)-\frac{\lambda}{2} R P^{2},
\end{aligned}
$$

where $R$ and $Q$ are, respectively, the curvilinear displacement coordinates for the $\mathrm{C}-\mathrm{H}$ stretch and for the bend, $P_{R}$ and $P$ are their conjugate momenta, $\mu$ is the reduced mass of the $\mathrm{C}-\mathrm{H}$ bond, $D$ and $a$ are the $\mathrm{C}-\mathrm{H}$ Morse parameters, $\lambda$ is a coupling constant, and $\omega_{2}^{0}$ is the zeroth order angular frequency of the bending mode. The constant $\lambda$ generally has a complicated dependence ${ }^{13,46(a)}$ on the atomic masses, equilibrium bond angles and lengths, and the bending normal coordinate ${ }^{47}$ coefficients $L_{i j}^{-1}$. This coupling constant will be treated as a variable parameter in the present paper.

For energies in the vicinity of the $|3,0\rangle$ state, it is assumed in this model that only one of the $\mathrm{C}-\mathrm{H}$ local mode vibrations need be considered. ${ }^{35}$ For these energies, the quantum mechanical eigenvalues for the symmetric and asymmetric combinations ${ }^{35}$ of local modes in the dihalomethanes become virtually degenerate, and hence the two CH's in the molecule have negligible direct coupling to each other.

The Fourier expansion for the displacement coordinate $R$ of a Morse oscillator is given in Ref. 48. Since the bending 
mode is a harmonic oscillator [cf. Eq. (5.1)], a suitable transformation to action-angle variables is to set $Q$ and $P$ equal to $\left(2 I_{2} / \omega_{2}^{0}\right)^{1 / 2} \sin \theta_{2}$ and $\left(2 I_{2} \omega_{2}^{o}\right)^{1 / 2} \cos \theta_{2}$, respectively. This transformation yields the $(1,-2)$ and $(0,0)$ Fourier components

$$
V_{1-2}\left(I_{1}, I_{2}\right)=\frac{I_{2} \omega_{2}^{0} \lambda}{4 a}\left(\frac{1-(1-y)^{1 / 2}}{y^{1 / 2}}\right)
$$

and

$$
V_{00}\left(I_{1}, I_{2}\right)=-\frac{I_{2} \omega_{2}^{0} \lambda}{2 a} \ln \left(\frac{1+(1-y)^{1 / 2}}{2(1-y)}\right) .
$$

where $y=E_{1}^{0} / D$, and $E_{1}^{0}$ and $D$ are the energy and dissociation energy of the zeroth order Morse oscillator, respectively. These expressions may be used in Eqs. (2.5), (2.6), (3.7), and (3.16).

\section{B. Calculations}

Calculations were performed for the resonant interaction of the $|3,0\rangle,|2,2\rangle$, and $|1,4\rangle$ states for the model Hamiltonian (5.1). The values used in Eqs. (5.1)-(5.3) for $\omega_{1}^{0}, \omega_{1}^{0} \chi, D$ and $a$, obtained from the data of of Ref. 35, were $3143,63.2,39076 \mathrm{~cm}^{-1}$, and 0.988 a.u., respectively. The zeroth order bend frequency $\omega_{2}^{0}$ was not determined there, but is estimated to be $\sim 1400 \mathrm{~cm}^{-1}$. The coupling parameter $\lambda$ was allowed to vary in the present calculations to yield quantum off-diagonal matrix elements between the zeroth order states $|3,0\rangle$ and $|2,2\rangle$ in the range of $5-30 \mathrm{~cm}^{-1}$. The resonant actions $I_{1}^{r}$ and $I_{2}^{r}$ used in evaluating the USC and two-state solutions were determined by the method described in Appendix A.

The Fermi resonant splittings and overlaps were calculated as a function of $\lambda$ using the three semiclassical methods presented in the previous sections. For the two-state and $3 \times 3$ semiclassical matrix treatment, calculations were performed using both the resonant and mean actions (cf. Sec. II) to evaluate the $3 \times 3$ semiclassical off-diagonal matrix elements and to obtain $q$ for the two-state calculation (Sec. IV A). In all cases, diagonalizations of quantum mechanical $2 \times 2,3 \times 3$, and $78 \times 78$ matrices were performed for comparison.

\section{Results}

The calculated splittings between the eigenstates of $|3,0\rangle$ and $|2,2\rangle$ parentage and between the eigenstates of $|3,0\rangle$ and $|1,4\rangle$ parentage are shown in Tables I and II,

TABLE I. Splittings (in $\left.\mathrm{cm}^{-1}\right)$ between states of $|3,0\rangle$ and $|2,2\rangle$ parentage.

\begin{tabular}{llllll}
\hline \hline$\lambda$ (a.u.) & $78 \times 78^{Q}$ & $3 \times 3^{Q}$ & $3 \times 3^{\text {sc }^{\mathrm{d}}}$ & $3 \times 3^{\mathrm{Sc}^{\mathrm{b}}}$ & USC $^{\mathrm{c}}$ \\
\hline 0.037 & 31.0 & 31.1 & 31.3 & 31.8 & 33.4 \\
0.077 & 30.8 & 31.1 & 31.9 & 33.5 & 35.5 \\
0.121 & 35.4 & 35.8 & 37.4 & 40.1 & 41.5 \\
0.170 & 43.1 & 43.8 & 46.1 & 49.2 & 49.7 \\
0.227 & 53.0 & 54.4 & 57.4 & 59.8 & 59.1 \\
\hline \hline
\end{tabular}

${ }^{\text {a }}$ Calculated with Fourier components [Eq. (2.6)] evaluated at mean actions for each pair of states.

${ }^{b}$ Calculated with Fourier components [Eq. (2.6)] evaluated at the resonant actions.

${ }^{c}$ Uniform semiclassical calculation (cf. Sec. IV B) based on $H_{\text {eff }}$ [Eq. (3.18)]
TABLE II. Splittings (in $\mathrm{cm}^{-1}$ ) between states of $|3,0\rangle$ and $|1,4\rangle$ parentage.

\begin{tabular}{lrrrrr}
\hline \hline$\lambda$ (a.u.) & $78 \times 78^{Q}$ & $3 \times 3^{Q}$ & $3 \times 3^{\mathrm{sc}^{\mathrm{c}}}$ & $3 \times 3^{\mathrm{sc}^{\mathrm{b}}}$ & $\mathrm{USC}^{\mathrm{c}}$ \\
\hline 0.037 & 61.9 & 62.3 & 62.2 & 61.2 & 64.1 \\
0.077 & 69.8 & 71.5 & 71.2 & 66.9 & 73.3 \\
0.121 & 78.0 & 82.2 & 81.7 & 71.9 & 82.3 \\
0.170 & 87.8 & 95.7 & 95.1 & 77.4 & 92.2 \\
0.227 & 100.4 & 113.8 & 113.2 & 84.1 & 103.1 \\
\hline \hline
\end{tabular}

${ }^{\mathrm{a}}$ Calculated with Fourier components [Eq. (2.6)] evaluated at mean actions for each pair of states.

${ }^{b}$ Calculated with Fourier components [Eq. (2.6)] evaluated at the resonant actions.

${ }^{\mathrm{c}}$ See footnote $\mathrm{c}$ of Table $\mathbf{I}$.

respectively. In Table III, the exact and the present uniform semiclassical (USC) results are compared with those obtained by the method of Ref. 7 (USC ${ }^{0}$ ). As in Ref. 7, the resonant actions for the $\mathrm{USC}^{0}$ calculation were found using the zeroth order frequencies, i.e., using $\mathrm{Eq}$. (A1) instead of Eq. (3.9). The "diagonal" perturbation term $V_{00}$ was then taken, in the USC ${ }^{0}$ method, as a constant value evaluated at these zeroth order resonant actions. Also shown in Table III, for comparison with and for analyzing the method of Ref. 7, are results from a quantum mechanical $3 \times 3$ matrix diagonalization method $\left(3 \times 3^{Q^{0}}\right)$ having the diagonal first order perturbation corrections taken as a constant (e.g., zero). In addition, the relative overlaps of the zeroth order states $\psi_{i}^{(0)}$ with the actual eigenstates $\psi_{j}$, defined as $\left|\left\langle\psi_{i}^{(0)} \mid \psi_{j}\right\rangle\right| /$ $\left|\left\langle\psi_{i}^{(0)} \mid \psi_{i}\right\rangle\right|$, are given in Table IV for two values of $\lambda$. For the various two-state calculations, the calculated splittings between the eigenstates of $|3,0\rangle$ and $|2,2\rangle$ parentage are given in Table $\mathrm{V}$ as a function of $\lambda$.

\section{DISCUSSION}

The quantum $3 \times 3$ and converged $(78 \times 78)$ results are seen from Tables I, II, and IV to be in good agreement (except at high $\lambda$ where the discrepancy in Table II is $\sim 10$ $\mathrm{cm}^{-1}$ ). Thus, the present Fermi resonance is well characterized by considering only the interaction of the three zeroth order states $|3,0\rangle,|2,2\rangle$, and $|1,4\rangle$. For the splittings, the $3 \times 3$ and USC semiclassical methods in Tables I and II yield results comparable to each other and in reasonable agree-

TABLE III. Calculated splittings (in $\mathrm{cm}^{-1}$ ) of $|3,0\rangle$ and $|2,2\rangle$ states by exact quantum, zero order uniform semiclassical (USC ${ }^{0}$ ), zero order $3 \times 3^{Q^{\mathrm{a}}}$ quantum, ${ }^{\mathrm{b}}$ and effective uniform semiclassical (USC) ${ }^{\mathrm{c}}$ methods.

\begin{tabular}{lllll}
\hline \hline$\lambda$ (a.u.) & $78 \times 78^{Q}$ & $3 \times 3^{Q^{\circ}}$ & $\begin{array}{l}\text { USC }^{0} \\
\text { [Ref. 25(a)] }\end{array}$ & $\begin{array}{l}\text { USC } \\
\text { (Present) }\end{array}$ \\
\hline 0.037 & 31.0 & 38.3 & 38.7 & 33.4 \\
0.077 & 30.8 & 44.4 & 45.7 & 35.5 \\
0.121 & 35.4 & 53.7 & 55.9 & 41.5 \\
0.170 & 43.1 & 65.7 & 68.1 & 49.7 \\
0.227 & 53.0 & 80.6 & $>80^{\mathrm{d}}$ & 59.1 \\
\hline \hline
\end{tabular}

${ }^{\text {a }}$ Using zeroth order frequencies [cf. Eq. (2.1)] to calculate $I_{1}^{r}, I_{2}^{r}, v$, and $q$.

${ }^{b}$ Calculated by setting all diagonal perturbations equal to zero in the $3 \times 3$ quantum matrix.

' See footnote $c$ of Table $I$.

d This value is estimated from the tables given in Ref. 41. The values of $q$ there go to 2.5 whereas $q$ equals 2.54 for the present $\mathrm{USC}^{\circ}$ method (with $\lambda=0.227$ ). 
TABLE IV. Relative overlaps. ${ }^{\mathrm{a}}$

\begin{tabular}{llllll}
\hline \hline$\lambda$ (a.u.) & $\begin{array}{l}\text { Rel. } \\
\text { overlap }^{\mathrm{a}}\end{array}$ & $78 \times 78^{Q}$ & $3 \times 3^{Q}$ & $3 \times 3^{\mathrm{sc}^{\mathrm{b}}}$ & USC $^{\mathrm{c}}$ \\
\hline 0.037 & & & & & \\
& $2: 3$ & 0.154 & 0.154 & 0.163 & 0.205 \\
& $1: 3$ & 0.008 & 0.008 & 0.008 & 0.007 \\
0.077 & $2: 1$ & 0.100 & 0.099 & 0.100 & 0.065 \\
& $2: 3$ & 0.347 & 0.344 & 0.359 & 0.507 \\
& $1: 3$ & 0.029 & 0.028 & 0.030 & 0.023 \\
& $2: 1$ & 0.189 & 0.186 & 0.186 & 0.111 \\
\hline \hline
\end{tabular}

${ }^{a}$ Relative overlap $j: i$ is defined as $\left|\left\langle\psi_{i}^{(0)} \mid \psi_{j}\right\rangle\right| /\left|\left\langle\psi_{i}^{(0)} \mid \psi_{i}\right\rangle\right|$, where $\left|\psi_{i, j}\right\rangle$ is the eigenstate of zeroth order $\left|\psi_{i \mid \lambda}^{(0)}\right\rangle$ parentage. States 3,2 , and 1 are defined as $|3,0\rangle,|2,2\rangle$, and $|1,4\rangle$, respectively.

${ }^{b}$ Calculated with Fourier components [Eq. (2.6)] evaluated using mean actions for each pair of states.

${ }^{\mathrm{c}}$ Present USC method (see footnote $c$ of Table I).

ment with the quantum mechanical values. The results shown in Tables I-V indicate that, of the semiclassical twostate, $3 \times 3$, and USC methods, the $3 \times 3$ one using mean actions in the evaluation of the Fourier components appears to be the most accurate for calculating both the splittings and the relative intensities. In addition, the results in Table III for the USC ${ }^{0}$ method of Ref. 7 show that the nonconstancy of the diagonal perturbation term $V_{00}$ has an appreciable effect for the system studied.

Of the two-state calculations, the quantum $2 \times 2$ values [based on the original Hamiltonian (5.1)] and the semiclassical two-state ones based on $H_{\text {eff }}$ [Eq. (3.18)] and employing mean actions in the calculation of the Fourier components are in better agreement than those based on $H_{\text {eff }}$ and resonant actions. Diagonalizations of semiclassical $2 \times 2$ matrices were also performed using the method of Sec. II. These results were essentially the same as those in Table $V$ based on $H_{\text {eff }}$ and mean actions. Thus, for the present model Hamiltonian (5.1), the two-state (Sec. IV A) and $2 \times 2$ (Sec. II) methods are essentially equivalent, although this is not necessarily the case in general.

The $3 \times 3$ (and $78 \times 78$ ) basis set calculations show that the $|1,4\rangle$ state mixes significantly with the other zeroth order states. Thus, the two-state treatments are incorrect for determining the eigenfunctions, and hence the overlaps (relative intensities), although they yield reasonable values for the eigenvalues (splittings) in Table V.

The semiclassical matrix calculations in Tables I-V indicate the principal weakness of the uniform approximation.

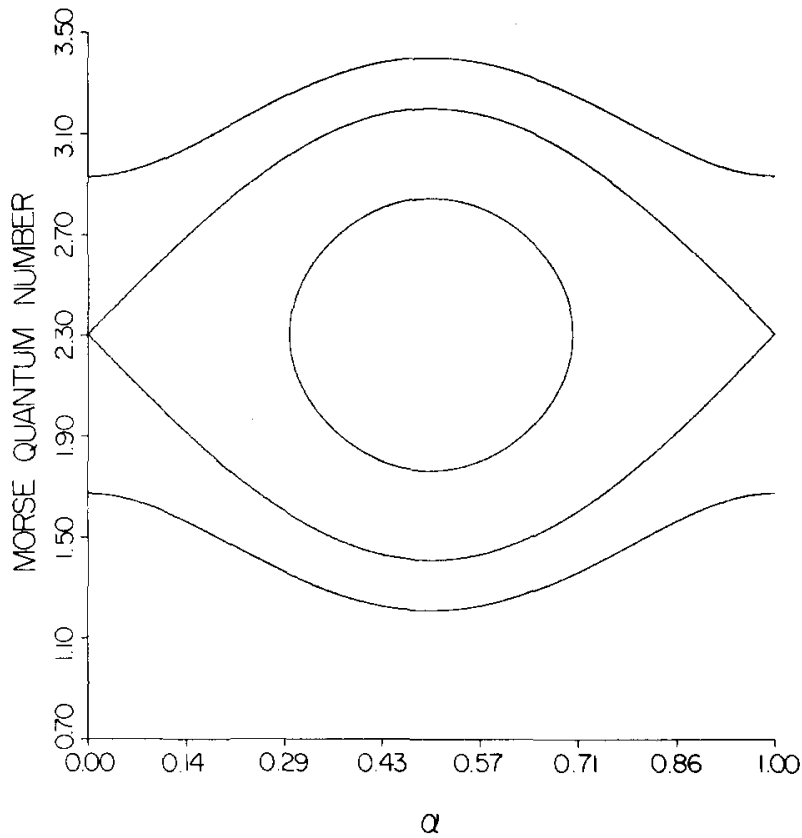

FIG. 1. An $\left(n_{1}, \alpha\right)$ phase plane portrait of the rotor Hamiltonian (B2) for the model Hamiltonian (5.1). The angle variable $\alpha$ is given in fractions of $\pi$.

In the derivation of the Mathieu equation and its corresponding solutions, $q$, and hence the $1,-2$ Fourier component, was approximated as a constant, i.e., it was evaluated at the resonance center. This approximation assumes, in effect, that all the matrix elements such as $\langle n, 0|V| n-1,2\rangle$ and $\langle n-1,2|V| n-2,4\rangle$ have the same average value. The semiclassical matrix technique employing mean actions for the evaluation of individual matrix elements $H_{i j}$ avoids this restriction of constant Fourier components and thus obtains better agreement with the purely quantum treatments in the general case. Nevertheless, the USC is useful because, when $q$ is small and expansions ${ }^{39(b)-39(c)}$ may be used for $a_{v}$ and $F(\alpha)$ of the Mathieu equation, it yields approximate analytic solutions. These solutions may also provide some additional physical insight when coupled with the classical analysis presented in Sec. III and Appendix B.

As an example of this latter point, one may consider the dependence of the Fermi resonances on the classical resonance width [Eq. (B3)]. Examination of the classical $\left(n_{\alpha}, \alpha\right)$ surfaces of section in Figs. 1 and 2 for the Hamiltonian (5.1) with $q=0.8$ shows that the states $|3,0\rangle$ and $|2,2\rangle$, with $n_{1}=3$ and $n_{1}=2$, are within the width of the resonance.

TABLE V. Splittings (in $\left.\mathrm{cm}^{-1}\right)$ between states of $|3,0\rangle$ and $|2,2\rangle$ parentage using two-state solutions.

\begin{tabular}{lllll}
\hline \hline$\lambda($ a.u. $)$ & $78 \times 78^{Q}$ & $2 \times 2^{Q}$ & Two-state $^{\text {sCa }}$ & Two-state $^{\text {scb }}$ \\
\hline 0.037 & 31.0 & 30.2 & 33.2 & 32.3 \\
0.077 & 30.8 & 28.4 & 35.4 & 32.3 \\
0.121 & 35.4 & 32.8 & 42.0 & 37.3 \\
0.170 & 43.1 & 43.1 & 51.5 & 47.0 \\
0.227 & 53.0 & 58.6 & 62.7 & 61.3 \\
\hline \hline
\end{tabular}

a Calculated using the method of Sec. IV A based on the effective Hamiltonian $H_{\text {eff }}$ [Eq. (3.18)] and using resonant actions in the evaluation of $q$ [Eq. (4.2)].

${ }^{b}$ Calculated using the method of Sec. IV A based on the effective Hamiltonian $H_{\text {eff }}$ [Eq. (3.18)] and using the mean actions between the states $|3,0\rangle$ and $|2,2\rangle$ in evaluating $q$ [Eq. (4.2)]. 


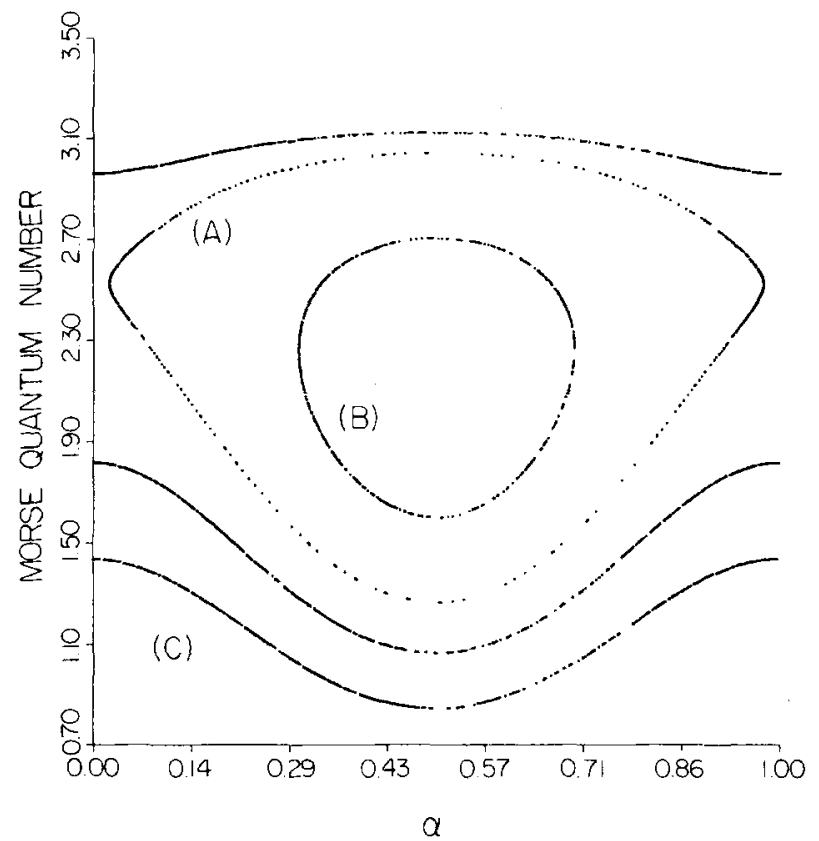

FIG. 2. An $\left(n_{1}, \alpha\right)$ surface of section for the Hamiltonian (5.1) taken for $P=0, Q>0$, and $\lambda=0.077$ a.u. $(q=0.8)$. Trajectories labeled $(\mathrm{A}),(\mathrm{B})$, and (C) are for initial condition corresponding to $n_{1}=3, n_{1}=2$, and $n_{1}=1$, respectively. All trajectories have $I_{\beta}=2 n_{1}+n_{2}+3 / 2=7.5$. The angle variable $\alpha$ is given in fractions of $\pi$.

Hence, one would expect significant mixing for these states, and this is indeed found to be the case (cf. Table IV for $\lambda=0.077)$. On the other hand, the state $|1,4\rangle$, with $n_{1}=1$, is well outside the classical resonance zone and does not mix strongly with the $|3,0\rangle$ or $|2,2\rangle$ states. One obtains similar conclusions about the degree of state mixing from purely quantum mechanical arguments by comparing the coupling elements $H_{i j}$ to the diagonal energy differences $E_{i}-E_{j}$. However, when many states are involved, the semiclassical phase plane picture allows one to estimate the degree of coupling by a single quantity, the width of the resonance [Eq. (B3)].

\section{CONCLUDING REMARKS}

The semiclassical methods presented in this paper all involve the use of the Fourier components of the perturbation, some of which exist in analytic form ${ }^{33,34,48}$ or are straightforward to evaluate in the typical case by numerical quadrature. For the calculation described in Sec. V, the semiclassical matrix elements (i.e., Fourier components) were analytic and could be evaluated by the use of a hand calculator. The quantum mechanical Morse matrix elements of, for instance $r$ or $r^{2}$, while analytic, ${ }^{49}$ are more complicated to compute. The semiclassical techniques can therefore be particularly useful when one wishes to use relatively simple methods for comparison with experimental absorption spectra: In actual experiments, the rovibrational structure may only be partially resolved (e.g., Ref. 35), and so a quick and approximate estimation of the Fermi resonance splittings and relative intensities can be helpful in fitting the data to various models.

\section{ACKNOWLEDGMENT}

This work was supported by a grant from the National Science Foundation.

\section{APPENDIX A: APPROXIMATE EVALUATION OF THE RESONANT ACTIONS}

The solution of Eqs. (3.9) and (3.12) to calculate the resonant actions $I_{1}^{r}$ and $I_{2}^{r}$ involves the determination of the angle-independent effective oscillator frequencies from Eqs. (3.5)-(3.7). One recalls that these frequencies are nonlinearly dependent on the resonant actions $I_{1}^{r}$ and $I_{2}^{r}$, so the following approximate root finding procedure was used to determine them: (1) A value for the zeroth order resonant action $I_{1}^{\prime \prime}$ was determined from the zeroth order resonance condition

$$
\omega_{1}^{0}-2 I r_{1}^{\circ} \omega_{1}^{0} \chi=2 \omega_{2}^{0},
$$

and $I_{2}^{\prime \prime}$ was found from Eq. (3.12) (one recalls that $I_{\beta}$ is taken as a constant of the motion). (2) The zeroth order values of $I_{1}^{\circ}$ and $I_{2}^{\prime \prime}$ were used to calculate the constants $g^{\prime \prime}$ and $f^{\prime \prime}$ from Eq. (3.7). (3) Approximate values for $I_{1}^{r}$ and $I_{2}^{r}$ were calculated from Eqs. (3.9) and (3.12) using $g^{\omega^{\circ}}$ and $f^{r^{2}}$ in determining the frequencies [Eqs. (3.5) and (3.6)] for the effective zeroth order Hamiltonian [Eq. (3.8)]. If desired, this procedure may then be iterated. However, for the model Hamiltonian given by Eq. (5.1), one iteration was sufficient to determine $I_{1}^{r}$ and $I_{2}^{r}$ to within $5 \%$ of the exact numerically calculated values and was the procedure used in the present paper.

\section{APPENDIX B: PHASE PLANE BEHAVIOR OF THE RESONANCE HAMILTONIAN}

The analysis of the $\left(I_{\alpha}, \alpha\right)$ phase plane behavior for the resonance Hamiltonian [Eq. (3.17)] is standard. For a given energy $E_{R}$ of the $\alpha$ motion in Eq. (3.17), $I_{\alpha}$ is given by

$$
I_{\alpha}=I_{\alpha}^{r} \pm 2\left[\Omega^{2}+\omega_{1} \chi\left(E_{R}-V_{0} \cos 2 \alpha\right)\right]^{1 / 2} / \omega_{1} \chi,
$$

where, from Eqs. (3.9), (3.10), (3.12), and (3.14), $I_{\alpha}^{r}$ denotes $2 \Omega / \omega_{1} \chi$. Since $I_{\alpha}=2 I_{1}$ and, semiclassically, $I_{1}=\left(n_{1}+\frac{1}{2}\right)$, a phase plane portrait for the Morse "quantum number" $n_{1}$ may be generated from $\mathrm{Eq}$. (B1) as a function of $\alpha$ :

$$
n_{1}=n_{1}^{r} \pm\left[\Omega^{2}+\omega_{1} \chi\left(E_{R}-V_{0} \cos 2 \alpha\right)\right]^{1 / 2} / \omega_{1} \chi,
$$

where $n_{1}^{r}$ is given by Eq. (3.19).

Figure 1 shows an $\left(n_{1}, \alpha\right)$ phase plane plot on the interval $(0, \pi)$ for the three different types of motion of Eq. (B2). This idealized behavior for the full Hamiltonian (2.1) is identical to that for a pendulum or "rotor" Hamiltonian. ${ }^{1-6}$ The curves that pass through a single point at $\alpha=0$ and $\pi$ correspond to the separatrix trajectory. The phase plane curves in Fig. 1 above and below the separatrix correspond to "rotations" in the $\left(n_{1}, \alpha\right)$ space. These are motions in which the action $I_{1}=I_{\alpha} / 2$ (or the quantum number $n_{1}=I_{1}-\frac{1}{2}$ ) varies only slightly over a cycle of motion. Thereby, there is relatively little classical energy transfer between the Morse and harmonic oscillators. The phase plane curves inside the separatrix represent motions in which $n_{1}$ varies greatly over a cycle of motion and thus reflects a large transfer of energy. Such a large variation in $n_{1}$ is expected for any initial $n_{1}$ within the resonance width

$$
\Delta n_{1}=\Delta I_{1}=2\left(2 V_{0} / \omega_{1} \chi\right)^{1 / 2}=2 \sqrt{q},
$$

defined by the separatrix trajectory. ${ }^{1-6}$ The width of the reso- 
nance increases with increasing coupling element $V_{0}$ and with decreasing effective anharmonicity $\omega_{1} \mathcal{X}$ of the Morse oscillator. The less the anharmonicity, the less the states in the progression $|n, 0\rangle,|n-1,2\rangle$, etc. pass out of resonance.

In Fig. 2, an $\left(n_{1}, \alpha\right)$ surface of section (e.g., Ref. 17) of the Hamiltonian (5.1) is shown for actual classical trajectories having initial conditions corresponding to $I_{\beta}=7.5$ and $n_{1}=1$ to 3.6. For this model Hamiltonian, this plot shows the "rotor" or "pendulum" behavior, although it is somewhat distorted from Fig. 1. For larger perturbations, the surface of section becomes increasingly distorted from the idealized behavior shown in Fig. 1 (cf. discussion in Ref. 25).

A few remarks on the rate of classical and quantum energy exchange among the oscillators are perhaps in order. For a classical resonance Hamiltonian [e.g., Eq. (3.17)], there is extensive classical energy exchange when the system is within the cosine well. $\left(I_{\alpha}\right.$ changes considerably during the latter motion.) The frequency of the oscillatory energy exchange is then obtained by expanding the $\cos 2 \alpha$ term about its minimum and is found to be porportional to the square root of the coefficient of the cosine term in Eq. (3.17) [i.e., it is proportional to the square root of the $(1,-2)$ Fourier component of the perturbation]. Quantum mechanically, this type of energy exchange is expected to be approached when the initial wave packet consists of many eigenstates. When there are only two or three states, as in the present analysis, the frequency of energy exchange between zeroth order states is, in the case of an exact zeroth order degeneracy, proportional to the coupling matrix elements between the states. In the semiclassical limit, these matrix elements correspond to the Fourier components of the cosine perturbation term in Eq. (3.17). As a result, the quantum energy exchange frequency may be thought of, in the case of an exact resonance, as being proportional to the coefficient rather than, as in the purely classical case, the square root of the coefficient of the cosine function. Thus, it is expected that, in any wave packet analysis, one must distinguish between the classical (i.e., many quantum states) and highly quantum (i.e., few quantum states) cases.

\section{APPENDIX C: NORMALIZATION OF THE WAVE FUNCTION (4.17)}

A normalized wave function $N_{n_{1}} \psi_{n_{1}}(\alpha)$ satisfies

$$
N_{n_{1}}^{2} \int_{0}^{n} \psi_{n_{1}}^{*}(\alpha) \psi_{n_{1}}(\alpha) d \alpha=1 .
$$

By truncating the expansion (4.17) at terms of order $q^{2}$ and using Eq. (C1), $N_{n_{1}}^{2}$ is found to be given from

$$
\begin{aligned}
\frac{1}{N_{n_{1}}^{2} \pi} \simeq 1 & +\frac{q^{2}}{16}\left[\frac{1}{\left[v\left(n_{1}\right)+1\right]^{2}}+\frac{1}{\left[v\left(n_{1}\right)-1\right]^{2}}\right] \\
& +\frac{q^{4}}{1024}\left[\frac{1}{\left[v\left(n_{1}\right)+1\right]^{2}\left[v\left(n_{1}\right)+2\right]^{2}}\right. \\
& \left.+\frac{1}{\left[v\left(n_{1}\right)-1\right]^{2}\left[v\left(n_{1}\right)-2\right]^{2}}\right]
\end{aligned}
$$

The normalization series in Eq. (C2) may be "slow" to converge for one of the semiclassical eigenstates $\psi_{j}(\alpha)$ involved in the resonance, an example being when $v\left(n_{1}\right) \pm m$ is small relative to $q^{2 m}$ for that state $(m=1,2)$. In that case, one can determine the other overlaps $\left\langle\psi_{n_{1}}^{(0)} \mid \psi_{j}\right\rangle$ of nonnegligible magnitude and then use the normalization condition for the Fermi resonance

$$
\sum_{j}\left|\left\langle\psi_{n_{1}}^{(0)} \mid \psi_{j}\right\rangle\right|^{2}=1
$$

to determine the absolute value of the unknown overlap. This approximation was tested in quantum mechanical calculations discussed in Sec. V and found to agree with the exact results in the system chosen to within $2 \%$. The semiclassical wave functions are not suitable for determining the overlaps with the zeroth order states when the normalization series (C2) does not converge for several of the semiclassical eigenstates.

'B. V. Chirikov, E. Heil, and A. M. Sessler, J. Stat. Phys. 3, 307 (1971); B. V. Chirikov, Phys. Rep. 52, 263 (1979).

${ }^{2}$ G. H. Walker and J. Ford, Phys. Rev. 188, 416 (1969).

${ }^{3}$ E. V. Shuryak, Sov. Phys. JETP 44, 1070 (1976).

${ }^{4}$ See the review in P. Brumer, Adv. Chem. Phys. 47, 201 (1981).

${ }^{5}$ See the review in M. Tabor, Adv. Chem. Phys. 46, 73 (1981).

${ }^{6}$ E. F. Jaeger and A. J. Lichtenberg, Ann. Phys. (NY) 71, 319 (1972).

${ }^{7}$ T. Uzer, Chem. Phys. Lett. 110, 356 (1984).

${ }^{8}$ E. Fermi, Z. Phys. 71, 250 (1931).

${ }^{9} \mathrm{~W}$. Kaye, Spectrochim. Acta 6, 257 (1954).

${ }^{10}$ These resonances have been observed, for example, in $\mathrm{CH}_{2} \mathrm{X}_{2}$ (with $\mathrm{X}=\mathrm{D}, \mathrm{F}, \mathrm{Cl}, \mathrm{Br}, \mathrm{I}$ ) [B. R. Henry and I. Hung, Chem. Phys. 29, 465 (1978), and Refs. 12, 35, and 44], $\mathrm{CHCl}_{3}$ and $\mathrm{CHBr}_{3}$ (Ref. 44), 1, 1, 2, 2-tetrachloroethane and 1, 1, 1, 1-tetrabromoethane [B. R. Henry and M. A. Mohammadi, Chem. Phys. 55, 385 (1981)], tetramethylsilicon, tetramethylgermanium, and tetramethyltin [B. R. Henry, M. A. Mohammadi, I. Hanazaki, and R. Nakagaki, J. Phys. Chem. 87, 4827 (1983)], CHD (Ref. $^{2}$ 12), neopentane [B. R. Henry, O. Sonnich Mortensen, W. F. Murphy, and D. A. C. Compton, J. Chem. Phys. 79, 2583 (1983)], and $\mathrm{CHF}_{3}$ [H. R. Dubal, M. Lewerenz, and M. Quack, Faraday Discuss. Chem. Soc. 75, 358 (1983); K. von Puttkamer, H. R. Dubal, and M. Quack, ibid. 75, 197 (1983), and references cited therein].

"G. A. Voth, R. A. Marcus, and A. H. Zewail, J. Chem. Phys. 81, 5494 (1984).

${ }^{12}$ J. W. Perry, D. J. Moll, A. Kuppermann, and A. H. Zewail, J. Chem. Phys. 82, 1195 (1985).

${ }^{13}$ E. L. Sibert III, W. P. Reinhardt, and J. T. Hynes, Chem. Phys. Lett. 92, 455 (1982); J. Chem. Phys. 81, 1115 (1984); E. L. Sibert III, J. T. Hynes, and W. P. Reinhardt, ibid. 81, 1135 (1984); E. L. Sibert III, Ph.D. thesis, University of Colorado, 1983; V. Buch, R. B. Gerber, and M. A. Ratner, J. Chem. Phys. 81, 3393 (1984).

${ }^{14}$ R. A. Marcus, Ann. NY Acad. Sci. 357, 169 (1980).

${ }^{15}$ G. M. Zaslavsky, Phys. Rep. 80, 157 (1981).

${ }^{16}$ K. G. Kay, J. Chem. Phys. 72, 5955 (1980).

${ }^{17}$ For reviews, see (a) D. W. Noid, M. L. Koszykowski, and R. A. Marcus, Annu. Rev. Phys. Chem. 32, 267 (1981); (b) S. A. Rice, Adv. Chem. Phys. 47, $117(1981)$.

${ }^{18}$ D. W. Noid and R. A. Marcus, J. Chem. Phys. 67, 559 (1977).

${ }^{19}$ D. W. Noid, M. L. Koszykowski, and R. A. Marcus, J. Chem. Phys. 71, 2864 (1979).

${ }^{20}$ C. Jaffe' and P. Brumer, J. Chem. Phys. 73, 5646 (1980).

${ }^{21}$ E. L. Sibert III, W. P. Reinhardt, and J. T. Hynes, J. Chem. Phys. 77, 3583 (1982).

${ }^{22}$ (a) E. J. Heller, E. B. Stechel, and M. J. Davis, J. Chem. Phys. 71, 4759 (1979); 73, 4720 (1980); (b) N. De Leon, M. J. Davis, and E. J. Heller, ibid. 80, $794(1984)$.

${ }^{23}$ E. L. Sibert III, J. T. Hynes, and W. P. Reinhardt, J. Chem. Phys. 77, 3595 (1982); J. S. Hutchinson, E. L. Sibert III, and J. T. Hynes, ibid. 81, 1314 (1984).

${ }^{24}$ D. W. Noid, M. L. Koszykowski, and R. A. Marcus, J. Chem. Phys. 78, $4018(1983)$.

${ }^{25}$ T. Uzer, D. W. Noid, and R. A. Marcus, J. Chem. Phys. 79, 4412 (1983); T. Uzer and R. A. Marcus, ibid. 81, 5013 (1984).

${ }^{26}$ R. T. Swimm and J. B. Delos, J. Chem. Phys. 71, 1706 (1979). 
${ }^{27}$ C. Jaffe and W. P. Reinhardt, J. Chem. Phys. 71, 1862 (1979); 77, 5191 (1982).

${ }^{28}$ The $I_{1}$ and $I_{2}$ in Eq. (2.1) are the usual action variables divided by $2 \pi$ [see, for example, H. Goldstein, Classical Mechanics (Addison-Wesley, Reading, Mass., 1980), p. 457].

${ }^{29}$ The derivation of the resonance Hamiltonian given in Ref. 7 is for a specific perturbation. For completeness, the derivation for a general perturbation is given in the present paper.

${ }^{30} \mathrm{~A}$ different treatment using semiclassical matrix elements is given in $\mathbf{R}$. B. Gerber and M. A. Ratner, Chem. Phys. Lett. 68, 195 (1979); R. B. Gerber, R. M. Roth, and M. A. Ratner, Mol. Phys. 44, 1335 (1981).

${ }^{31}$ R. A. Marcus, Chem. Phys. Lett. 7, 525 (1970).

${ }^{32}$ For the case of coupled states having widely varying quantum numbers, use of the geometric mean for the intermediate actions in the evaluation of the semiclassical matrix elements [Eq. (2.6)] may yield somewhat more accurate results [for example, P. F. Naccache, J. Phys. B 5, 1308 (1972) and Ref. 34]. The arithmetic mean is not well-suited for use in the semiclassical matrix elements $H_{i j}$ when the difference in quantum numbers between states $i$ and $j$ is large (Ref. 34).

${ }^{33}$ M. L. Koszykowski, D. W. Noid, and R. A. Marcus, J. Phys. Chem. 86, 2113 (1982).

${ }^{34}$ D. M. Wardlaw, D. W. Noid, and R. A. Marcus, J. Phys. Chem. 88, 536 (1984); D. M. Wardlaw, D. W. Noid, and R. A. Marcus (to be published).

${ }^{35}$ O. Sonnich Mortensen, B. R. Henry, and M. A. Mohammadi, J. Chem. Phys. 75, 4800 (1981).

${ }^{36}$ M. L. Sage, J. Phys. Chem. 83, 1455 (1979).

${ }^{37}$ The resonance Hamiltonian [Eq. (3.17)] differs from the usual resonance Hamiltonian (for example, Refs. 1-6) by the term linear in $I_{\alpha}$ and the $\cos 2 \alpha$ (instead of $\cos \alpha$ ) angle dependence. This $2 \alpha$ angle term is introduced to obtain the standard Mathieu equation in Eq. (4.12).

${ }^{38}$ J. C. Duncan, D. Ellis, and I. J. Wright, Mol. Phys. 20, 673 (1971).

${ }^{39}$ (a) N. W. McLachlan, Theory and Applications of Mathieu Functions (Clarendon, Oxford, 1947); (b) Expansion (6) in Sec. 2.16 of Ref. 39(a); (c) $F(\alpha)=A c e_{v}(\alpha, q)+B s e_{v}(\alpha, q)$ from Sec. 2.16 of Ref. 39(a), where $A=1$ and $B=i$. The restriction $v>0$ is unnecessary.
${ }^{40}$ To determine the value of $v_{i}$, one uses the zeroth order primitive wave functions (Refs. 25 and 31) as a guide. Since a solution to the zeroth order Mathieu equation (when $q=0$ ) is $\sim \exp [i v \alpha]$, the corresponding zeroth order wave function [Eq. $(4.11)]$ is $\sim \exp [i(\xi-1+v) \alpha]$. On the other hand, the primitive semiclassical wave function is $\sim \exp \left[i n_{\alpha} \alpha\right]$. We therefore have $n_{a}$ equal to $\xi-1+v$, and hence the order $v$ is given by $2 n_{1}+1-\xi$. Upon introducing the expression given in Eq. (4.2) for $\xi$, one obtains Eq. (4.16) of the text for $v$. The notation $v\left(n_{1}\right)=2\left(n_{1}-n_{1}^{r}\right)$ may be used to distinguish between the different possible values of $v$ as a function of $n_{1}$. From Eq. (4.16), it is seen that the solutions $F(\alpha)$ of the Mathieu equation [Eq. (4.12)] are in general of fractional order [Ref. 39(a)].

${ }^{4 i}$ T. Tamir, Math. Comp. 16, 100 (1962).

${ }^{42}$ Handbook of Mathematical Functions, edited by M. Abramowitz and I. A. Stegun (Dover, New York, 1965), Chap. 20.

${ }^{43}$ J. N. L. Connor, T. Uzer, R. A. Marcus, and A. D. Smith, J. Chem. Phys. 80, 5095 (1984).

${ }^{44}$ H. L. Fang and R. L. Swofford, J. Chem. Phys. 72, 6382 (1980).

${ }^{45}$ For the two states involved in an avoided crossing, the orders $v_{1}$ and $v_{2}$ of the Mathieu equation [Eq. (4.12)] become integers (Ref. 25). The appropriate solutions are then Mathieu functions of integer order [Ref. 39(a)]. Using these functions, one would proceed as in Sec. IV B to obtain suitable overlap formulas. Since the avoided crossing point is such a special condition, a treatment of the wave function for this case has been omitted in the interests of brevity.

${ }^{46}$ (a) E. L. Sibert III, J. T. Hynes, and W. P. Reinhardt, J. Phys. Chem. 87, 2032 (1983); (b) L. A. Gribov, Opt. Spectrosc. 31, 842 (1971); H. M. Pickett, J. Chem. Phys. 56, 1715 (1972); R. Meyer and H. H. Gunthard, ibid. 49, 1510 (1968); C. R. Quade, ibid. 64, 2783 (1976); 79, 4089 (1983); W. B. Clodius and C. R. Quade, ibid. 80, 3528 (1984).

${ }^{47}$ E. B. Wilson, Jr., J. C. Decius, and P. C. Cross, Molecular Vibrations (Dover, New York, 1955), Chap. 4.

${ }^{48}$ I. E. Sazonov and N. I, Zhirnov, Opt. Spectrosc. 34, 254 (1973).

${ }^{49}$ M. L. Sage, Chem. Phys. 35, 375 (1978); J. A. C. Gallas, Phys. Rev. A 21, 1829 (1980); V. S. Vasan and R. J. Cross, J. Chem. Phys. 78, 3869 (1983). 